\title{
Differential Space-Time Block Code Modulation for DS-CDMA Systems
}

\author{
Jianhua Liu \\ Department of Electrical and Computer Engineering, P.O. Box 116130, University of Florida, Gainesville, FL 32611, USA \\ Email: jhliu@dsp.ufl.edu \\ Jian Li \\ Department of Electrical and Computer Engineering, P.O. Box 116130, University of Florida, Gainesville, FL 32611, USA \\ Email: li@dsp.ufl.edu
}

\begin{abstract}
Erik G. Larsson
Department of Electrical and Computer Engineering, P.O. Box 116130, University of Florida, Gainesville, FL 32611, USA Email: larsson@dsp.ufl.edu
\end{abstract}

Received 29 May 2001 and in revised form 27 December 2001

\begin{abstract}
A differential space-time block code (DSTBC) modulation scheme is used to improve the performance of DS-CDMA systems in fast time-dispersive fading channels. The resulting scheme is referred to as the differential space-time block code modulation for DS-CDMA (DSTBC-CDMA) systems. The new modulation and demodulation schemes are especially studied for the down-link transmission of DS-CDMA systems. We present three demodulation schemes, referred to as the differential space-time block code Rake (D-Rake) receiver, differential space-time block code deterministic (D-Det) receiver, and differential space-time block code deterministic de-prefix (D-Det-DP) receiver, respectively. The D-Det receiver exploits the known information of the spreading sequences and their delayed paths deterministically besides the Rake type combination; consequently, it can outperform the D-Rake receiver, which employs the Rake type combination only. The D-Det-DP receiver avoids the effect of intersymbol interference and hence can offer better performance than the D-Det receiver.
\end{abstract}

Keywords and phrases: wireless communications, space-time coding, smart antennas, spread spectrum, DS-CDMA, Rake receiver.

\section{INTRODUCTION}

The performance of wireless communications systems can be increased drastically by exploiting the spatial diversities offered by multiple transmit and receive antennas in fading channels [1]. Traditionally, research work in spatial diversity mainly focused on the receiving diversity. In fact, receive antenna diversity is being used in base stations to improve the reception in current cellular systems, whereas transmit diversity is just beginning to attract attention [1].

Recently, Alamouti [2] proposed a simple and useful transmit diversity scheme to improve the overall performance of wireless communication systems in flat fading channels. This scheme is one of many interesting techniques emerging in the field of space-time coding, a new coding and signal processing technique that is designed for use with multiple transmit antennas (cf. [1] and the references therein). It introduces temporal and spatial correlation into signals transmitted from different antennas to provide transmit diversity and coding gain over an uncoded system. However, Alamouti's method as well as many other transmit diversity schemes, such as those in $[3,4]$, are based on the assumption that perfect channel state information (CSI) is available at the receiver. Although training is a feasible way to obtain CSI when the channel is stationary or changes slowly, it will incur excessive overhead or even fail when the channel experiences fast fading. (Third-generation European cellular standards are required to operate on trains with a speed of up to $500 \mathrm{~km} / \mathrm{h}$ [5].) In view of this, differential space-time modulation (DSTM) schemes were introduced as extensions of the traditional differential phase shift keying (DPSK) [6] in flat fading channels $[5,7,8]$. DSTM schemes obviate the need for channel estimation at the receiver, while maintaining the desired properties of space-time coding techniques. By combining the merits of the DSTM scheme and the spread spectrum technology, a differential space-code modulation (DSCM) scheme was devised to combat unknown interference and jamming in flat fading channels [9]. 
More recently, space-time block codes (STBC) based differential space-time block code (DSTBC) modulation scheme was proposed in [10]. DSTBC has the same diversity gain as but offers higher coding gain than the unitary group code based DSTM schemes. This is not surprising in view of the fact that STBC is optimal in terms of SNR [11]. Moreover, the decoding of the DSTBC modulation scheme is more efficient than for the DSTM schemes, especially for large constellations, since the former allows for a decoupled linear detection, with which it is to detect each information symbol separately.

In this paper, we combine the merits of DSTBC and the spread spectrum technology to propose a new modulation scheme to improve the performance of DS-CDMA systems in fast time-dispersive fading channels. This scheme is referred to as the differential space-time block code modulation for DS-CDMA (DSTBC-CDMA) systems. The new modulation and demodulation schemes are studied for the down-link synchronous transmission of DS-CDMA systems with multiple antennas at both the base station and mobile units, operating in fast time-dispersive fading channels. We devise three demodulation schemes, referred to as the differential spacetime block code Rake (D-Rake) receiver, differential spacetime block code deterministic (D-Det) receiver, and differential space-time block code deterministic de-prefix (D-Det-DP) receiver. By making use of a two-step least-square (LS) fitting approach, the proposed modulation and demodulation schemes yield simple receivers.

We demonstrate with simulation examples that the DDet receiver can perform better than the D-Rake receiver in that the former can offer lower bit-error-rate (BER), especially for moderate to high SNR. The reason is that the D-Rake receiver employs the Rake type combination only, whereas the D-Det receiver not only employs the Rake type combination, but also exploits the known information of spreading sequences and their delayed paths deterministically. Since the D-Det-DP receiver avoids the effect of intersymbol interference by discarding the data samples corrupted by the intersymbol interference, it can offer better performance than the D-Det receiver. We note that the superiority of the D-Det and D-Det-DP receivers over the D-Rake receiver is not due to the number of transmit antennas; this means that even when we have only one transmit antenna, using every spreading sequence including all of their delayed versions deterministically is still a better choice than the Rake type receiver.

The rest of the paper is organized as follows. Section 2 briefly reviews the DSTBC modulation scheme. Section 3 describes the DSTBC-CDMA scheme. Three demodulation approaches, namely the D-Rake, D-Det, and D-Det-DP receivers, are given in Section 4. Simulation results are presented in Section 5. Finally, we deliver our comments and conclusions in Section 6.

\section{REVIEW OF DSTBC}

To facilitate our presentation, we first briefly review the DSTBC modulation scheme of [10]. Let $M, N, L$, and $P$ denote the number of transmit antennas, the number of receive antennas, the time length of a space-time block code, and the number of symbols transmitted during $L$ time samples, respectively. Let $\mathbf{C}_{k}$ be the $k$ th $M \times L$ space-time block code matrix to be transmitted by the $M$ antennas over $L$ time samples (transmitted column by column). For simplicity, we only consider the case of $M=L$ (see [10] for more general cases). In this case, $\mathbf{C}_{k}$ satisfies

$$
\mathbf{C}_{k}^{H} \mathbf{C}_{k}=\mathbf{C}_{k} \mathbf{C}_{k}^{H}=\mathbf{I}_{M},
$$

where $(\cdot)^{H}$ denotes the conjugate transpose, $\mathbf{I}_{M}$ is an $M \times M$ identity matrix, and

$$
\mathbf{C}_{k}=\mathbf{C}_{k-1} \mathbf{G}_{k}, \quad \mathbf{C}_{0}=\mathbf{I}_{M},
$$

with $\mathbf{G}_{k}$ being the $k$ th $M \times M$ information carrying matrix. Let $\bar{s}_{k, p}, \tilde{s}_{k, p}, p=1,2, \ldots, P$, be, respectively, the real and imaginary parts of $s_{k, p}$, the $p$ th information symbol of the time block $k$, which is an element of a unitary constellation $\mathscr{C}$ (i.e., $\left|s_{k, p}\right|^{2}=1, p=1,2, \ldots, P$ ). We have

$$
\mathbf{G}_{k}=\frac{1}{P}\left(\sum_{p=1}^{P} \mathbf{A}_{p} \bar{s}_{k, p}+i \sum_{p=1}^{P} \mathbf{B}_{p} \tilde{s}_{k, p}\right),
$$

where $\mathbf{A}_{p}, \mathbf{B}_{p}, p=1,2, \ldots, P$, are the space-time coding matrices obtained from the theory of orthogonal designs and amicable orthogonal designs $[12,13]$, which have the following properties:

$$
\begin{array}{rlrl}
\mathbf{A}_{j}^{H} \mathbf{A}_{j} & =\mathbf{A}_{j} \mathbf{A}_{j}^{H}=\mathbf{I}, & \mathbf{B}_{j}^{H} \mathbf{B}_{j}=\mathbf{B}_{j} \mathbf{B}_{j}^{H}=\mathbf{I}, & \forall j, \\
\mathbf{A}_{j}^{H} \mathbf{A}_{k} & =-\mathbf{A}_{k}^{H} \mathbf{A}_{j}, & \mathbf{A}_{j} \mathbf{A}_{k}^{H}=-\mathbf{A}_{k} \mathbf{A}_{j}^{H}, & j \neq k, \\
\mathbf{B}_{j}^{H} \mathbf{B}_{k}=-\mathbf{B}_{k}^{H} \mathbf{B}_{j}, & \mathbf{B}_{j} \mathbf{B}_{k}^{H}=-\mathbf{B}_{k} \mathbf{B}_{j}^{H}, & j \neq k, \\
\mathbf{A}_{j}^{H} \mathbf{B}_{k}=\mathbf{B}_{k}^{H} \mathbf{A}_{j}, & \mathbf{A}_{j} \mathbf{B}_{k}^{H}=\mathbf{B}_{k} \mathbf{A}_{j}^{H}, & \forall k, j .
\end{array}
$$

It can be shown that [10]

$$
\mathbf{G}_{k} \mathbf{G}_{k}^{H}=\mathbf{G}_{k}^{H} \mathbf{G}_{k}=\mathbf{I}
$$

when the above properties hold. The data rate is

$$
R=\frac{P}{L} \log _{2}|\mathscr{G}| \quad \mathrm{bit} / \mathrm{s} / \mathrm{Hz},
$$

where $|\mathscr{C}|$ denotes the number of elements in $\mathscr{C}$. For example, for $M=L=P=2$,

$$
\begin{aligned}
\mathbf{A}_{1} & =\left[\begin{array}{ll}
1 & 0 \\
0 & 1
\end{array}\right], & \mathbf{A}_{2} & =\left[\begin{array}{cc}
0 & 1 \\
-1 & 0
\end{array}\right], \\
\mathbf{B}_{1} & =\left[\begin{array}{ll}
0 & 1 \\
1 & 0
\end{array}\right], & \mathbf{B}_{2} & =\left[\begin{array}{cc}
1 & 0 \\
0 & -1
\end{array}\right],
\end{aligned}
$$

is a set of matrices satisfying the above properties. Note that this set of matrices represents the Alamouti code [2].

Let $\mathbf{H} \in \mathbb{C}^{N \times M}$ be the fading matrix in a flat fading environment and let $\mathbf{E}_{k} \in \mathbb{C}^{N \times L}$ be the additive noise matrix. The array received data matrix $\mathbf{Y}_{k} \in \mathbb{C}^{N \times L}$ has the form 


$$
\mathbf{Y}_{k}=\mathbf{H C}_{k}+\mathbf{E}_{k}
$$

where $\mathbf{H}$ is assumed to be a deterministic unknown matrix and the elements of $\mathbf{E}_{k}$ are assumed to be independently and identically distributed complex Gaussian random variables with zero-mean and variance $\sigma^{2}$. The channel fading matrix $\mathbf{H}$ is assumed to be the same for $\mathbf{Y}_{k-1}$ and $\mathbf{Y}_{k}$. Then the maximum likelihood (ML) differential receiver is given by [10]

$$
\begin{aligned}
\hat{s}_{k, p}=\arg \max _{s_{k, p} \in \mathcal{C}}\{ & \operatorname{Re}\left[\operatorname{tr}\left(\mathbf{A}_{p}^{H} \mathbf{Y}_{k-1}^{H} \mathbf{Y}_{k}\right) \bar{s}_{k, p}\right] \\
- & \left.\operatorname{Re}\left[\operatorname{tr}\left(i \mathbf{B}_{p}^{H} \mathbf{Y}_{k-1}^{H} \mathbf{Y}_{k}\right) \tilde{s}_{k, p}\right]\right\}, \quad p=1,2, \ldots, P,
\end{aligned}
$$

where $\operatorname{Re}\{\cdot\}$ denotes the real part of the argument and $\operatorname{tr}(\cdot)$ denotes the trace of a matrix. Note that the detection of the $P$ symbols $\left\{s_{k, p}\right\}_{p=1}^{P}$ is decoupled.

\section{DSTBC-CDMA MODULATION}

The above DSTBC modulation scheme can be readily modified for the down-link synchronous transmission of DS-CDMA systems, where multiple transmit antennas are easy to deploy at base stations. In view of this, we consider the down-link case only in this paper though the schemes proposed herein can be applied to the up-link case as well.

Let $U$ be the number of synchronous down-link users, and $\mathbf{C}_{k, u} \in \mathbb{C}^{M \times L}$ be the space-time block code matrix for user $u, u=1,2, \ldots, U$, at the $k$ th time block (the time interval used to transmit $U$ space-time matrices simultaneously). By using $L$ spreading sequences to spread the $L$ columns of $\mathrm{C}_{k, u}$ (to transmit the $L$ columns simultaneously) for each user and by using different spreading sequences for different users (to transmit the space-time codes of all of the users together), we have the new modulation scheme-DSTBC-CDMA. At the $j$ th time sample of the $k$ th time block, the transmitted signal is

$$
\mathbf{s}_{k}(j)=\sum_{u=1}^{U} \mathbf{C}_{k, u} \mathbf{d}_{u}(j), \quad j=1,2, \ldots, J,
$$

where $J$ is the length of the spreading sequences and $\left\{\mathbf{d}_{u}(j) \in\right.$ $\left.\mathbb{C}^{L \times 1}, j=1,2, \ldots, J\right\}$ are the $L$ unit-energy spreading sequences for the $u$ th user. (Note that we do not constrain the spreading sequences to be short ones and hence this scheme is also applicable to long sequences.) The information carrying matrix

$$
\mathbf{G}_{k, u}=\frac{1}{P}\left(\sum_{p=1}^{P} \mathbf{A}_{p} \bar{s}_{k, u, p}+i \sum_{p=1}^{P} \mathbf{B}_{p} \tilde{s}_{k, u, p}\right) \in \mathbb{C}^{L \times L}
$$

is related to $\mathbf{C}_{k, u}$ and $\mathbf{C}_{k-1, u}$ as follows:

$$
\mathbf{C}_{k, u}=\mathbf{C}_{k-1, u} \mathbf{G}_{k, u}, \quad u=1,2, \ldots, U .
$$

We consider herein the case that each user knows the spreading sequences of all other users; the confidentiality of the different users can be done by other means such as encryption. We consider time-dispersive fast fading channels by modeling the channel impulse response as a finite impulse response (FIR) filter with length $L_{f}[14]$ and assume that the code-timing is known. Let $\mathbf{y}_{k, l_{f}}(j)$ denote the $j$ th sample of the receiver output (noise free) of the $l_{f}$ th tap of the FIR filter, $l_{f}=0,1, \ldots, L_{f}-1$, at the $k$ th time block. Then

$$
\mathbf{y}_{k, l_{f}}(j)=\sum_{u=1}^{U} \mathbf{H}_{l_{f}} \mathbf{C}_{k, u} \mathbf{d}_{u}\left(j-l_{f}\right), \quad l_{f}=0,1, \ldots, L_{f}-1,
$$

where $\mathbf{H}_{l_{f}} \in \mathbb{C}^{N \times M}$ is the deterministic unknown channel matrix of the $l_{f}$ th tap between the $N$ receive antennas and the $M$ transmit antennas. Note that all users have the same channel in the down-link transmission. The output of the receiver antenna is

$$
\begin{aligned}
\mathbf{y}_{k}(j) & =\sum_{l_{f}=0}^{L_{f}-1} \mathbf{y}_{k, l_{f}}(j)+\mathbf{e}_{k}(j) \\
& \triangleq \sum_{l_{f}=0}^{L_{f}-1} \mathbf{H}_{l_{f}} \mathbf{C}_{k} \mathbf{d}\left(j-l_{f}\right)+\mathbf{e}_{k}(j), \quad j=1,2, \ldots, J,
\end{aligned}
$$

where $\mathbf{C}_{k}=\left[\begin{array}{llll}\mathbf{C}_{k, 1} & \mathbf{C}_{k, 2} & \cdots & \mathbf{C}_{k, U}\end{array}\right] \in \mathbb{C}^{M \times L U}, \mathbf{d}(j)=$ $\left[\begin{array}{llll}\mathbf{d}_{1}^{T}(j) & \mathbf{d}_{2}^{T}(j) & \cdots & \mathbf{d}_{U}^{T}(j)\end{array}\right]^{T} \in \mathbb{C}^{L U \times 1}$ with $(\cdot)^{T}$ denoting the transpose, and $\mathbf{e}_{k}(j)$ denotes the additive zero-mean white complex Gaussian noise vector with covariance matrix $\sigma^{2} \mathbf{I}$ plus the intersymbol interference (ISI) due to transmitting $\mathrm{C}_{k-1}$.

\section{DEMODULATION SCHEMES}

We obtain below three different demodulation schemes, that is, the D-Rake, D-Det, and D-Det-DP receivers for the symbol detection.

\subsection{The D-Rake receiver}

By employing the tap-delay expression, we can stack up the $L_{f}$ vector outputs of (14) into a larger vector as

$$
\begin{aligned}
& \mathbf{x}_{k}(j) \triangleq\left[\begin{array}{c}
\mathbf{y}_{k}(j) \\
\mathbf{y}_{k}(j+1) \\
\vdots \\
\mathbf{y}_{k}\left(j+L_{f}-1\right)
\end{array}\right] \\
&=\left[\begin{array}{c}
\mathbf{H}_{0} \mathbf{C}_{k} \mathbf{d}(j)+\tilde{\mathbf{e}}_{k, 0}(j) \\
\mathbf{H}_{1} \mathbf{C}_{k} \mathbf{d}(j)+\tilde{\mathbf{e}}_{k, 1}(j) \\
\vdots \\
\mathbf{H}_{L_{f}-1} \mathbf{C}_{k} \mathbf{d}(j)+\tilde{\mathbf{e}}_{k, L_{f}-1}(j)
\end{array}\right] \in \mathbb{C}^{N L_{f} \times 1}, \\
& j=1,2, \ldots, J,
\end{aligned}
$$

where for $\tilde{l}_{f}=0,1, \ldots, L_{f}-1$,

$$
\tilde{\mathbf{e}}_{k, \tilde{l}_{f}}(j)=\sum_{l_{f}=0, l_{f} \neq \tilde{l}_{f}}^{L_{f}-1} \mathbf{H}_{l_{f}} \mathbf{C}_{k} \mathbf{d}\left(j-l_{f}+\tilde{l}_{f}\right)+\mathbf{e}_{k}\left(j+\tilde{l}_{f}\right) .
$$


In (16), we have lumped all but the $\tilde{l}_{f}$ th path of each signal into $\tilde{\mathbf{e}}_{k, \tilde{l}_{f}}(j)$. Note that for some $j$ and $\tilde{l}_{f}, \tilde{\mathbf{e}}_{k, \tilde{l}_{f}}(j)$ also contains the tails of the previous modulated space-time codes $\mathbf{C}_{k-1}$ or the heads of the following modulated space-time codes $\mathbf{C}_{k+1}$.

Let

$$
\mathbf{H}=\left[\begin{array}{llll}
\mathbf{H}_{0}^{T} & \mathbf{H}_{1}^{T} & \cdots & \mathbf{H}_{L_{f}-1}^{T}
\end{array}\right]^{T} \in \mathbb{C}^{N L_{f} \times M}
$$

and $\tilde{\mathbf{e}}_{k}(j)=\left[\begin{array}{llll}\tilde{\mathbf{e}}_{k, 0}^{T}(j) & \tilde{\mathbf{e}}_{k, 1}^{T}(j) & \cdots & \tilde{\mathbf{e}}_{k, L_{f}-1}^{T}(j)\end{array}\right]^{T} \in \mathbb{C}^{N L_{f} \times 1}$. We have

$$
\mathbf{x}_{k}(j)=\mathbf{H C}_{k} \mathbf{d}(j)+\tilde{\mathbf{e}}_{k}(j)
$$

By denoting

$$
\begin{aligned}
\mathbf{X}_{k} & =\left[\begin{array}{llll}
\mathbf{x}_{k}(1) & \mathbf{x}_{k}(2) & \cdots & \mathbf{x}_{k}(J)
\end{array}\right] \in \mathbb{C}^{N L_{f} \times J}, \\
\mathbf{D} & =\left[\begin{array}{llll}
\mathbf{d}(1) & \mathbf{d}(2) & \cdots & \mathbf{d}(J)
\end{array}\right] \in \mathbb{C}^{L U \times J}, \\
\tilde{\mathbf{E}}_{k} & =\left[\begin{array}{llll}
\tilde{\mathbf{e}}_{k}(1) & \tilde{\mathbf{e}}_{k}(2) & \cdots & \tilde{\mathbf{e}}_{k}(J)
\end{array}\right] \in \mathbb{C}^{N L_{f} \times J},
\end{aligned}
$$

we have the following data matrix:

$$
\mathbf{X}_{k}=\mathbf{H C}_{k} \mathbf{D}+\tilde{\mathbf{E}}_{k} .
$$

By assuming that the channel matrix $\mathbf{H}$ remains constant while transmitting two adjacent space-time codes $\mathbf{C}_{k-1}$ and $\mathrm{C}_{k}$ for all users, we consider the LS approach for the detection of $\left\{s_{k, u, p}\right\}_{u=1, \ldots, U, p=1, \ldots, p}$. Since the noise and interference term $\tilde{\mathbf{E}}_{k}$ is not white, the LS fitting approach is different from the exact ML estimator, which is hard to obtain. Consider first the direct use of the LS fitting approach:

$$
\begin{aligned}
& \left\{\hat{s}_{k, u, p}\right\}_{u=1, \ldots, U, p=1, \ldots, P} \\
& =\arg \min _{\left\{s_{k, u, p}\right\}_{u=1, \ldots,, p=1, \ldots, P}}\left\|\left[\mathbf{X}_{k-1} \mathbf{X}_{k}\right]-\mathbf{H}\left[\begin{array}{ll}
\mathbf{C}_{k-1} & \mathbf{C}_{k}
\end{array}\right]\left(\mathbf{I}_{2} \otimes \mathbf{D}\right)\right\|_{F}^{2},
\end{aligned}
$$

where $\otimes$ denotes the Kronecker matrix product [15] and $\|\cdot\|_{F}$ is the Frobenius norm. For this case, we could concentrate out $\mathbf{H}$ and then minimize the cost function with respect to $\left\{s_{k, u, p}\right\}_{u=1, \ldots, U, p=1, \ldots, P}$. However, the detection of $\left\{s_{k, u, p}\right\}_{u=1, \ldots, U, p=1, \ldots, P}$ would not be decoupled and hence would require an exhaustive search, which is impractical when $U$ and $P$ are large. To decouple the detection of $\left\{s_{k, u, p}\right\}_{u=1, \ldots, U, p=1, \ldots, P}$, let

$$
\mathbf{F}_{k} \triangleq \mathbf{H}\left[\begin{array}{ll}
\mathbf{C}_{k-1} & \mathbf{C}_{k}
\end{array}\right] .
$$

We first determine the estimate $\hat{\mathbf{F}}_{k}$ of $\mathbf{F}_{k}$ by using the following LS fitting approach:

$$
\hat{\mathbf{F}}_{k}=\arg \min _{\mathbf{F}_{k}}\left\|\left[\begin{array}{ll}
\mathbf{X}_{k-1} & \mathbf{X}_{k}
\end{array}\right]-\mathbf{F}_{k}\left(\mathbf{I}_{2} \otimes \mathbf{D}\right)\right\|_{F^{\prime}}^{2}
$$

which gives

$$
\hat{\mathbf{F}}_{k}=\left[\begin{array}{lll}
\mathbf{X}_{k-1} \mathbf{D}^{\dagger} & \mathbf{X}_{k} \mathbf{D}^{\dagger}
\end{array}\right]
$$

where $\mathbf{D}^{\dagger}=\mathbf{D}^{H}\left(\mathbf{D D}^{H}\right)^{-1}$ is the pseudo inverse of $\mathbf{D}$. Note that we have used the assumption that $\mathbf{D D}^{H}$ is of full rank (a necessary condition for this is that $L U<J$ ) in the above derivation, which can be guaranteed by controlling the number of users in the DSTBC-CDMA systems.

Next, by exploiting the structure of $\mathbf{F}_{k}$, a second LS fitting leads to

$$
\begin{aligned}
& \left\{\hat{s}_{k, u, p}\right\}_{u=1, \ldots, U, p=1, \ldots, P} \\
& \quad=\arg \min _{\left\{s_{k, u, p}\right\}_{u=1, \ldots, U, p=1, \ldots, P}}\left\|\hat{\mathbf{F}}_{k}-\mathbf{H}\left[\begin{array}{ll}
\mathbf{C}_{k-1} & \mathbf{C}_{k}
\end{array}\right]\right\|_{F}^{2},
\end{aligned}
$$

which renders a simple decoupled D-Rake receiver for each user (see the appendix for a detailed derivation):

$$
\begin{aligned}
& \hat{s}_{k, u, p}=\arg \max _{s_{k, u, p} \in \mathcal{C}}\{ \operatorname{Re}\left[\operatorname{tr}\left(\mathbf{A}_{p}^{H} \tilde{\mathbf{F}}_{k-1, u}^{H} \tilde{\mathbf{F}}_{k, u}\right) \bar{s}_{k, u, p}\right] \\
&\left.-\operatorname{Re}\left[\operatorname{tr}\left(i \mathbf{B}_{p}^{H} \tilde{\mathbf{F}}_{k-1, u}^{H} \tilde{\mathbf{F}}_{k, u}\right) \tilde{s}_{k, u, p}\right]\right\}, \\
& u=1,2, \ldots, U, p=1,2, \ldots, P,
\end{aligned}
$$

where $\hat{\mathbf{F}}_{k} \triangleq\left[\begin{array}{ll}\tilde{\mathbf{F}}_{k-1} & \tilde{\mathbf{F}}_{k}\end{array}\right]$ with $\tilde{\mathbf{F}}_{k-1}$ and $\tilde{\mathbf{F}}_{k} \in \mathbb{C}^{N L_{f} \times L U}$ and

$$
\tilde{\mathbf{F}}_{k} \triangleq\left[\begin{array}{lll}
\tilde{\mathbf{F}}_{k, 1} & \cdots & \tilde{\mathbf{F}}_{k, U}
\end{array}\right],
$$

with $\tilde{\mathbf{F}}_{k, u} \in \mathbb{C}^{N L_{f} \times L}, u=1,2, \ldots, U$, being the submatrix corresponding to the $u$ th user.

\subsection{The D-Det receiver}

Equation (14) can be rewritten as

$$
\begin{aligned}
\mathbf{y}_{k}(j) & =\sum_{l_{f}=0}^{L_{f}-1} \mathbf{H}_{l_{f}} \mathbf{C}_{k} \mathbf{d}\left(j-l_{f}\right)+\mathbf{e}_{k}(j) \\
& \triangleq \overline{\mathbf{H}}\left(\mathbf{I}_{L_{f}} \otimes \mathbf{C}_{k}\right) \overline{\mathbf{d}}(j)+\mathbf{e}_{k}(j), \quad j=1,2, \ldots, J,
\end{aligned}
$$

where

$$
\begin{aligned}
& \overline{\mathbf{H}}=\left[\begin{array}{llll}
\mathbf{H}_{0} & \mathbf{H}_{1} & \cdots & \mathbf{H}_{L_{f}-1}
\end{array}\right] \in \mathbb{C}^{N \times M L_{f}}, \\
& \overline{\mathbf{d}}(j)=\left[\begin{array}{c}
\mathbf{d}(j) \\
\mathbf{d}(j-1) \\
\vdots \\
\mathbf{d}\left(j-L_{f}+1\right)
\end{array}\right] \in \mathbb{C}^{L U L_{f} \times 1} .
\end{aligned}
$$

By collecting the received data into a matrix, we have

$$
\begin{aligned}
\mathbf{Y}_{k} & =\left[\begin{array}{llll}
\mathbf{y}_{k}(1) & \mathbf{y}_{k}(2) & \cdots & \mathbf{y}_{k}(J)
\end{array}\right] \\
& =\overline{\mathbf{H}}\left(\mathbf{I}_{L_{f}} \otimes \mathbf{C}_{k}\right) \overline{\mathbf{D}}+\mathbf{E}_{k} \in \mathbb{C}^{N \times J},
\end{aligned}
$$

where $\overline{\mathbf{D}}=\left[\begin{array}{llll}\overline{\mathbf{d}}(1) & \overline{\mathbf{d}}(2) & \ldots & \overline{\mathbf{d}}(J)\end{array}\right] \in \mathbb{C}^{L U L_{f} \times J}$ and $\mathbf{E}_{k}=$ $\left[\begin{array}{llll}\mathbf{e}_{k}(1) & \mathbf{e}_{k}(2) & \cdots & \left.\mathbf{e}_{k}(J)\right] \in \mathbb{C}^{N \times J} .\end{array}\right.$

Let $\mathbf{Z}_{k}=\overline{\mathbf{H}}\left[\mathbf{I}_{L_{f}} \otimes \mathbf{C}_{k-1} \quad \mathbf{I}_{L_{f}} \otimes \mathbf{C}_{k}\right]$. Similar to (23) and (24), the following LS fitting approach:

$$
\hat{\mathbf{Z}}_{k}=\arg \min _{\mathbf{Z}_{k}}\left\|\left[\begin{array}{ll}
\mathbf{Y}_{k-1} & \mathbf{Y}_{k}
\end{array}\right]-\mathbf{Z}_{k}\left(\mathbf{I}_{2} \otimes \overline{\mathbf{D}}\right)\right\|_{F}^{2}
$$


leads to

$$
\hat{\mathbf{Z}}_{k}=\left[\begin{array}{ll}
\mathbf{Y}_{k-1} \overline{\mathbf{D}}^{\dagger} & \mathbf{Y}_{k} \overline{\mathbf{D}}^{\dagger}
\end{array}\right] \triangleq\left[\begin{array}{ll}
\check{\mathbf{Z}}_{k-1} & \check{\mathbf{Z}}_{k}
\end{array}\right],
$$

with $\check{\mathbf{Z}}_{k-1}$ and $\check{\mathbf{Z}}_{k} \in \mathbb{C}^{N \times L U L_{f}}$. We have assumed that $\overline{\mathbf{D}} \overline{\mathbf{D}}^{H}$ is of full rank (the necessary condition is that $L U L_{f}<J$ ) in getting (32).

Let

$$
\check{\mathbf{Z}}_{k}=\left[\begin{array}{llll}
\tilde{\mathbf{Z}}_{k, 0} & \tilde{\mathbf{Z}}_{k, 1} & \cdots & \tilde{\mathbf{Z}}_{k, L_{f}-1}
\end{array}\right],
$$

with $\tilde{\mathbf{Z}}_{k, l_{f}} \in \mathbb{C}^{N \times L U}, l_{f}=0,1, \ldots, L_{f}-1$, and

$$
\tilde{\mathbf{Z}}_{k}=\left[\begin{array}{c}
\tilde{\mathbf{Z}}_{k, 0} \\
\tilde{\mathbf{Z}}_{k, 1} \\
\vdots \\
\tilde{\mathbf{Z}}_{k, L_{f}-1}
\end{array}\right] \in \mathbb{C}^{N L_{f} \times L U}
$$

We have

$$
\begin{aligned}
& \left\{\hat{s}_{k, u, p}\right\}_{u=1, \ldots, U, p=1, \ldots, P} \\
& =\arg \min _{\left\{s_{k, u, p}\right\}_{u=1, \ldots, U, p=1, \ldots, P}} \|\left[\begin{array}{ll}
\check{Z}_{k-1} & \check{Z}_{k}
\end{array}\right] \\
& -\overline{\mathbf{H}}\left[\mathbf{I}_{L_{f}} \otimes \mathbf{C}_{k-1} \quad \mathbf{I}_{L_{f}} \otimes \mathbf{C}_{k}\right] \|_{F}^{2} \\
& =\arg \min _{\left\{s_{k, u, p}\right\}_{u=1, \ldots, U, p=1, \ldots, P}}\left\|\left[\begin{array}{ll}
\tilde{\mathbf{Z}}_{k-1} & \tilde{\mathbf{Z}}_{k}
\end{array}\right]-\mathbf{H}\left[\begin{array}{ll}
\mathbf{C}_{k-1} & \mathbf{C}_{k}
\end{array}\right]\right\|_{F}^{2} \text {, }
\end{aligned}
$$

which is similar to (25).

Similar to (26), a simple D-Det receiver is given by

$$
\begin{array}{r}
\hat{s}_{k, u, p}=\arg \max _{s_{k, u, p} \in \mathscr{C}}\left\{\operatorname{Re}\left[\operatorname{tr}\left(\mathbf{A}_{p}^{H} \tilde{\mathbf{Z}}_{k-1, u}^{H} \tilde{\mathbf{Z}}_{k, u}\right) \bar{s}_{k, u, p}\right]\right. \\
\left.-\operatorname{Re}\left[\operatorname{tr}\left(i \mathbf{B}_{p}^{H} \tilde{\mathbf{Z}}_{k-1, u}^{H} \tilde{\mathbf{Z}}_{k, u}\right) \tilde{s}_{k, u, p}\right]\right\}, \\
u=1,2, \ldots, U, p=1,2, \ldots, P .
\end{array}
$$

where $\tilde{\mathbf{Z}}_{k, u}$ is the submatrix in $\tilde{\mathbf{Z}}_{k}$ corresponding to user $u$ defined in the same way as for $\tilde{\mathbf{F}}_{k, u}$ shown in (27).

Note that besides the Rake type combination of the delayed versions from each path, the D-Det receiver exploits the known information of the spreading sequences as well as their delayed paths deterministically. Hence the D-Det receiver outperforms the D-Rake receiver, especially for moderate to high SNR. Note also that the full rank assumption for $\overline{\mathbf{D}} \overline{\mathbf{D}}^{H}$ seems to imply that the D-Det receiver intrinsically has lower subscriber processing capacity than the D-Rake receiver. Yet this drawback is not serious at all since DS-CDMA systems usually work in quite light load conditions [16]. (We will see in Section 5 that when $J=63$ and $M=L=2$, even for $U$ as small as 7 (using 14 Gold sequences), the BER curve for the D-Rake receiver will become flat at a relatively low SNR, which means that it is nearly fully loaded and cannot accommodate more users.)

\subsection{The D-Det-DP receiver}

Note that the noise and ISI term $\mathbf{E}_{k}$ in (30) is not temporally white since the first $L_{f}-1$ columns contain the tails of the modulated space-time codes $\mathbf{C}_{k-1}$. When the SNR is high, the ISI will dominate $\mathbf{E}_{k}$. We could add a prefix (also called a guard interval) to separate two consecutive space-time codes. In the demodulation, we simply need to de-prefix (discard) the prefix (the data in the guard interval) before demodulation. A similar approach, which we use herein, is to simply discard the first $L_{f}-1$ columns of $\mathbf{Y}_{k}$ to obtain $\breve{\mathbf{Y}}_{k}$, where

$$
\begin{aligned}
\breve{\mathbf{Y}}_{k} & =\left[\begin{array}{llll}
\mathbf{y}_{k}\left(L_{f}\right) & \mathbf{y}_{k}\left(L_{f}+1\right) & \cdots & \mathbf{y}_{k}(J)
\end{array}\right] \\
& =\overline{\mathbf{H}}\left(\mathbf{I}_{L_{f}} \otimes \mathbf{C}_{k}\right) \breve{\mathbf{D}}+\breve{\mathbf{E}}_{k} \in \mathbb{C}^{N \times\left(J-L_{f}+1\right)}
\end{aligned}
$$

with $\breve{\mathbf{D}}=\left[\begin{array}{llll}\overline{\mathbf{d}}\left(L_{f}\right) & \overline{\mathbf{d}}\left(L_{f}+1\right) & \ldots & \overline{\mathbf{d}}(J)\end{array}\right] \in \mathbb{C}^{L U L_{f} \times\left(J-L_{f}+1\right)}$ and $\breve{\mathbf{E}}_{k}=\left[\begin{array}{llll}\mathbf{e}_{k}\left(L_{f}\right) & \mathbf{e}_{k}\left(L_{f}+1\right) & \cdots & \mathbf{e}_{k}(J)\end{array}\right] \in \mathbb{C}^{N \times\left(J-L_{f}+1\right)}$. This deprefix approach works especially well if $L_{f} \ll J$ since the information loss is very slight. Note that $\breve{\mathbf{E}}_{k}$ is the white noise and hence the direct use of LS fitting gives the ML detector.

To decouple the detection of $\left\{s_{k, u, p}\right\}_{u=1, \ldots, U, p=1, \ldots, P}$ like in the $\mathrm{D}$-Det receiver, we have the $\mathrm{D}$-Det-DP receiver as

$$
\begin{aligned}
& \hat{s}_{k, u, p}=\arg \max _{s_{k, u, p} \in \mathscr{C}}\{ \operatorname{Re}\left[\operatorname{tr}\left(\mathbf{A}_{p}^{H} \breve{\mathbf{Z}}_{k-1, u}^{H} \breve{\mathbf{Z}}_{k, u}\right) \bar{s}_{k, u, p}\right] \\
&\left.-\operatorname{Re}\left[\operatorname{tr}\left(i \mathbf{B}_{p}^{H} \breve{\mathbf{Z}}_{k-1, u}^{H} \breve{\mathbf{Z}}_{k, u}\right) \tilde{s}_{k, u, p}\right]\right\}, \\
& u=1,2, \ldots, U, p=1,2, \ldots, P .
\end{aligned}
$$

where $\breve{\mathbf{Z}}_{k, u}$ is the submatrix in $\breve{\mathbf{Z}}_{k}$ corresponding to user $u$ defined in the same way as for $\tilde{\mathbf{F}}_{k, u}$ shown in (27) and

$$
\begin{gathered}
\breve{\mathbf{Z}}_{k}=\left[\begin{array}{c}
\grave{\mathbf{Z}}_{k, 0} \\
\grave{\mathbf{Z}}_{k, 1} \\
\vdots \\
\grave{\mathbf{Z}}_{k, L_{f}-1}
\end{array}\right], \\
\grave{\mathbf{Z}}_{k} \triangleq \breve{\mathbf{Y}}_{k} \breve{\mathbf{D}}^{\dagger} \triangleq\left[\begin{array}{llll}
\grave{\mathbf{Z}}_{k, 0} & \grave{\mathbf{Z}}_{k, 1} & \cdots & \grave{\mathbf{Z}}_{k, L_{f}-1}
\end{array}\right],
\end{gathered}
$$

with $\grave{\mathbf{Z}}_{k, l_{f}} \in \mathbb{C}^{N \times L U}, l_{f}=0,1, \ldots, L_{f}$. Again, we need $\breve{\mathbf{D}}^{H}$ to be of full rank (the necessary condition of which is $L U L_{f}<$ $\left.J-L_{f}+1\right)$.

Note that the D-Det-DP receiver avoids the effect of ISI by discarding the data samples corrupted by ISI and hence, as we will see in the simulation examples of Section 5, can offer better performance than the D-Det receiver.

\section{NUMERICAL EXAMPLES}

We present several numerical examples to demonstrate the performance of the proposed D-Rake, D-Det, and D-Det-DP receivers for DSTBC-CDMA systems. Two sets of examples are given in this section: D-Rake, D-Det, and D-Det-DP receivers for time-invariant channels as well as for time-varying channels. 


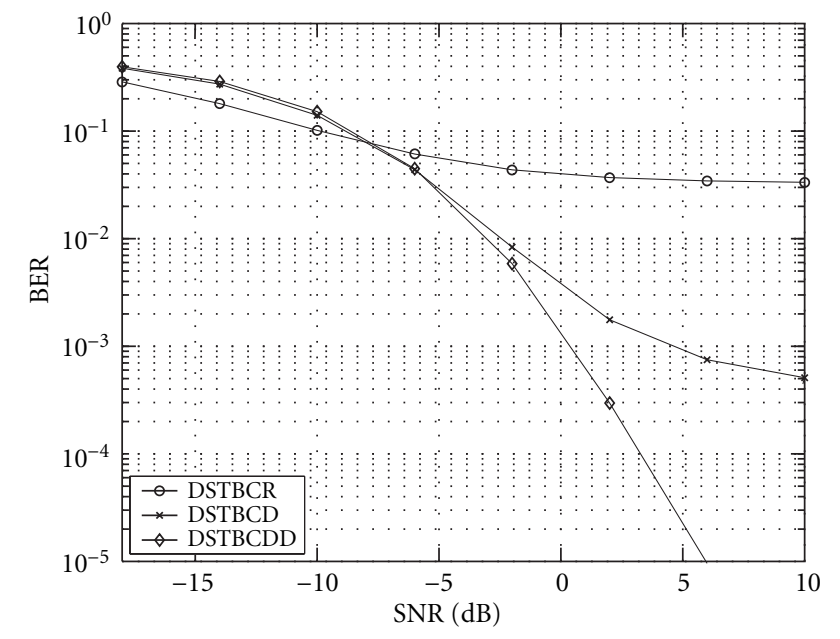

FIgURe 1: BER versus SNR comparison for D-Rake, D-Det, and DDet-DP receivers for time-invariant fading channels when $U=7$.

The DSTBC-CDMA based communications system considered herein is equipped with $M=2$ transmit antennas and $N=1$ receive antenna and employs the $2 \times 2$ space-time coding matrices, as described by (7). QPSK constellation is used herein; hence $R=2 \mathrm{bits} / \mathrm{sec} / \mathrm{Hz}$. Unit-energy Gold sequences with length $J=63$ are used as spreading sequences in all of the simulations. We choose $L_{f}=3$ and generate the channel vectors (channel matrices $\mathbf{H}_{l_{f}}$ becomes channel vectors $\mathbf{h}_{l_{f}}$ since $N=1)$ according to $\mathbf{h}_{l_{f}}^{H} \sim \mathcal{N}\left(0, \rho_{l_{f}} \mathbf{I}_{2}\right), l_{f}=0,1,2$, with $\rho_{l_{f}}$ being the signal power of the $l_{f}$ th tap. The attenuation between two consecutive taps is assumed to be $3 \mathrm{~dB}$. Note that the SNR used herein, which is defined according to tap 0 for each user, is given by

$$
\tilde{\rho}=\frac{\rho_{0}}{J \sigma^{2}} .
$$

When the time-varying fading is considered, the channel vectors $\mathbf{h}_{l_{f}}, l_{f}=0,1,2$, change from one time sample to another, according to the Jakes' model [17].

\subsection{D-Rake, D-Det, and D-Det-DP receivers for time-invariant channels}

Figure 1 shows the BER comparison for D-Rake, D-Det, and D-Det-DP receivers as a function of the SNR for timeinvariant fading channels when $U=7$. Note that the D-Det receiver performs better than the D-Rake receiver, especially for moderate to high SNR. The reason is that the D-Det receiver not only employs a kind of combination similar to the Rake receiver, but also exploits the known information of the spreading sequences and their delayed versions deterministically, whereas the D-Rake receiver employs the Rake type combination only. We also note that the D-Det-DP receiver performs better than the D-Det receiver due to avoiding ISI, which dominates the noise and ISI term when the SNR is high.

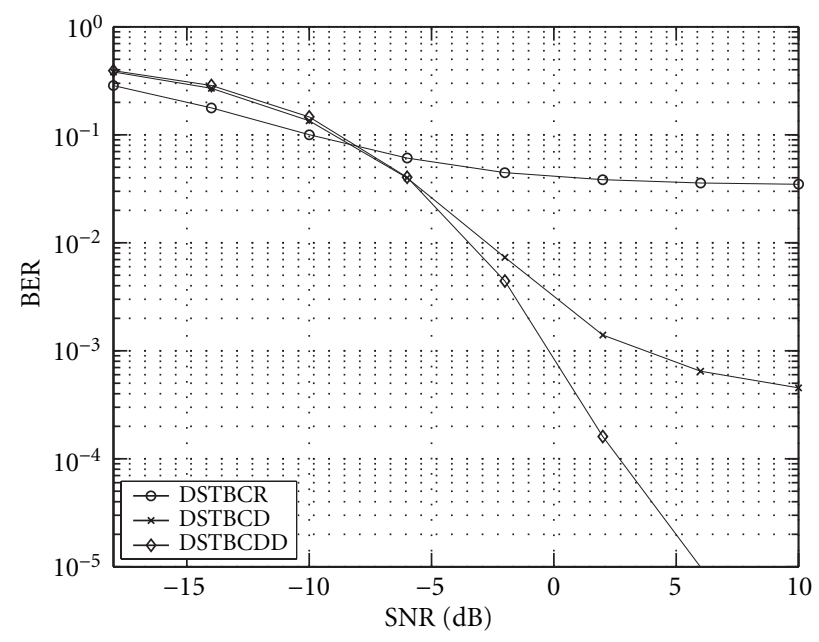

FIGURe 2: BER versus SNR comparison for D-Rake, D-Det, and DDet-DP receivers for time-varying fading channels when $U=7$.

\subsection{D-rake, D-Det, and D-Det-DP receivers for time-varying channels}

The simulated channels in the following case experience time-varying fading risen from the Doppler frequencies caused by the relative motions of mobiles and/or surroundings. It is characterized by the normalized Doppler frequency of $f_{D} T_{c}$, where $f_{D}$ is the Doppler frequency and $T_{c}$ is the chip duration of the spreading sequences. In the simulations, we choose $1 / T_{c}=1.2288 \mathrm{MHz}$, and $f_{D}=200 \mathrm{~Hz}$ (corresponding to a vehicle moving at $71 \mathrm{mph}$ (miles per hour) with the carrier frequency being $1.9 \mathrm{GHz}$ ).

Figure 2 shows the BER comparison for D-Rake, D-Det, and D-Det-DP receivers as a function of the SNR for timevarying fading channels when $U=7$. Again, the $\mathrm{D}$-Det receiver performs better than the D-Rake receiver for moderate to high SNR and the D-Det-DP receiver performs better than the D-Det receiver. Note also that the demodulation schemes are quite robust against the (fast) timing-varying of fading.

\section{CONCLUSIONS}

We have proposed a new spatial and temporal modulation scheme, referred to as the differential space-time block code modulation for DS-CDMA (DSTBC-CDMA) systems. The new modulation and demodulation schemes are especially studied for the down-link transmission for DS-CDMA systems operating in fast time-dispersive fading channels. We have devised three demodulation schemes, referred to as the differential space-time block code Rake (D-Rake) receiver, differential space-time block code deterministic (D-Det) receiver, and differential space-time block code deterministic de-prefix $(D-D e t-D P)$ receiver. Simulation results have shown that the $\mathrm{D}$-Det receiver is superior to the D-Rake receiver in that the former offers lower BER for moderate to high SNR due to exploiting the known information of the spreading sequences and their delayed paths deterministically in addition to the 
Rake type combination. The D-Det-DP receiver offers better performance than the D-Det receiver due to avoiding ISI. Moreover, the superiority of D-Det and D-Det-DP receivers over the D-Rake receiver is not due to the number of transmit antennas and should be a better choice than the Rake type receiver for the case of only one transmit antenna as well.

\section{APPENDIX}

\section{DERIVATION OF THE DSTBC-CDMA RECEIVER}

In this appendix, we give a detailed derivation of the DSTBC-CDMA receiver based on the following LS fitting approach:

$$
\begin{aligned}
& \left\{\hat{s}_{k, u, p}\right\}_{u=1, \ldots, U, p=1, \ldots, P} \\
& \quad=\arg \min _{\left\{s_{k, u, p}\right\}_{u=1, \ldots, U, p=1, \ldots, P}}\left\|\left[\begin{array}{ll}
\mathbf{Y}_{k-1} & \mathbf{Y}_{k}
\end{array}\right]-\mathbf{H}\left[\begin{array}{ll}
\mathbf{C}_{k-1} & \mathbf{C}_{k}
\end{array}\right]\right\|_{F}^{2},
\end{aligned}
$$

which is the general form of (25) and (35). By partitioning $\mathbf{Y}_{k}$ into submatrices corresponding to each user, (A.1) becomes

$$
\begin{aligned}
& \left\{\hat{s}_{k, u, p}\right\}_{u=1, \ldots, U, p=1, \ldots, P} \\
& =\arg \min _{\left\{s_{k, u, p}\right\}_{u=1, \ldots, U, p=1, \ldots, P}} \sum_{u=1}^{U} \|\left[\begin{array}{ll}
\mathbf{Y}_{k-1, u} & \mathbf{Y}_{k, u}
\end{array}\right] \\
& -\mathbf{H}\left[\begin{array}{ll}
\mathbf{C}_{k-1, u} & \mathbf{C}_{k, u}
\end{array}\right] \|_{F}^{2},
\end{aligned}
$$

which readily decouples the demodulation as

$$
\begin{array}{r}
\left\{\hat{s}_{k, u, p}\right\}_{p=1}^{P} \\
=\arg \min _{\left\{s_{k, u, p}\right\}_{p=1}^{P} \in \mathscr{C}}\left\|\left[\begin{array}{ll}
\mathbf{Y}_{k-1, u} & \mathbf{Y}_{k, u}
\end{array}\right]-\mathbf{H}\left[\begin{array}{ll}
\mathbf{C}_{k-1, u} & \mathbf{C}_{k, u}
\end{array}\right]\right\|_{F}^{2}, \\
u=1,2, \ldots, U .
\end{array}
$$

For notational simplicity, we drop the subscripts $k$ and $u$ in deriving the DSTBC-CDMA receiver for user $u$ in the $k$ th time block below and get

$$
\begin{aligned}
&\left\{\hat{s}_{p}\right\}_{p=1}^{P}=\arg \min _{\left\{s_{p}\right\}_{p=1}^{P} \in \mathscr{C}} \| {\left[\begin{array}{ll}
\mathbf{Y}_{k-1} & \mathbf{Y}_{k}
\end{array}\right]-\mathbf{H C}_{k-1}\left[\begin{array}{ll}
\mathbf{I} & \mathbf{G}_{k}
\end{array}\right] \|_{F}^{2} } \\
&=\arg \min _{\left\{s_{p}\right\}_{p=1}^{P} \in \mathscr{C}} \operatorname{tr}\{\left(\left[\begin{array}{ll}
\mathbf{Y}_{k-1} & \mathbf{Y}_{k}
\end{array}\right]-\mathbf{H C}_{k-1}\left[\begin{array}{ll}
\mathbf{I} & \mathbf{G}_{k}
\end{array}\right]\right) \\
&\left.\times\left(\left[\begin{array}{ll}
\mathbf{Y}_{k-1} & \mathbf{Y}_{k}
\end{array}\right]-\mathbf{H C}_{k-1}\left[\begin{array}{ll}
\mathbf{I} & \mathbf{G}_{k}
\end{array}\right]\right)^{H}\right\} .
\end{aligned}
$$

Since $\mathbf{H}$ is assumed to be a deterministic unknown matrix, $\mathbf{H}$ can be concentrated out as

$$
\begin{aligned}
\hat{\mathbf{H}} & =\left[\begin{array}{ll}
\mathbf{Y}_{k-1} & \mathbf{Y}_{k}
\end{array}\right]\left[\begin{array}{c}
\mathbf{I} \\
\mathbf{G}_{k}^{H}
\end{array}\right] \mathbf{C}_{k-1}^{H}\left(\mathbf{C}_{k-1}\left[\begin{array}{ll}
\mathbf{I} & \mathbf{G}_{k}
\end{array}\right]\left[\begin{array}{c}
\mathbf{I} \\
\mathbf{G}_{k}^{H}
\end{array}\right] \mathbf{C}_{k-1}^{H}\right)^{-1} \\
& =\frac{1}{2 L}\left[\begin{array}{ll}
\mathbf{Y}_{k-1} & \mathbf{Y}_{k}
\end{array}\right]\left[\begin{array}{c}
\mathbf{I} \\
\mathbf{G}_{k}^{H}
\end{array}\right] \mathbf{C}_{k-1}^{H},
\end{aligned}
$$

where we have used the fact that $\mathbf{G}_{k} \mathbf{G}_{k}^{H}=\mathbf{I}$ and $\mathbf{C}_{k} \mathbf{C}_{k}^{H}=\mathbf{I}$. By inserting (A.5) into (A.4), we have

$$
\begin{aligned}
&\left\{\hat{s}_{p}\right\}_{p=1}^{P}=\arg \min _{\left\{s_{p} p_{p=1}^{P} \in \mathscr{C}\right.} \operatorname{tr}\{ \\
& {\left[\begin{array}{ll}
\mathbf{Y}_{k-1} & \mathbf{Y}_{k}
\end{array}\right]\left[\begin{array}{c}
\mathbf{Y}_{k-1}^{H} \\
\mathbf{Y}_{k}^{H}
\end{array}\right] } \\
&\left.-\frac{1}{2}\left[\begin{array}{ll}
\mathbf{Y}_{k-1} & \mathbf{Y}_{k}
\end{array}\right]\left[\begin{array}{c}
\mathbf{I} \\
\mathbf{G}_{k}^{H}
\end{array}\right]\left[\begin{array}{ll}
\mathbf{I} & \mathbf{G}_{k}
\end{array}\right]\left[\begin{array}{c}
\mathbf{Y}_{k-1}^{H} \\
\mathbf{Y}_{k}^{H}
\end{array}\right]\right\} .
\end{aligned}
$$

Hence, neglecting the irrelevant terms yields the following estimate [10]:

$$
\begin{aligned}
&\left\{\hat{s}_{p}\right\}_{p=1}^{P}= \arg \min _{\left\{s_{p}\right\}_{p=1}^{P} \in \mathscr{C}} \operatorname{tr}\left\{\left[\mathbf{Y}_{k-1} \quad \mathbf{Y}_{k}\right]\left[\begin{array}{cc}
\mathbf{I} & \mathbf{G}_{k} \\
\mathbf{G}_{k}^{H} & \mathbf{I}
\end{array}\right]\left[\begin{array}{c}
\mathbf{Y}_{k-1}^{H} \\
\mathbf{Y}_{k}^{H}
\end{array}\right]\right\} \\
&= \arg \max _{\left\{s_{p}\right\rangle_{p=1}^{P} \in \mathscr{C}} \operatorname{Re} \operatorname{tr}\left\{\mathbf{G}_{k}^{H} \mathbf{Y}_{k-1}^{H} \mathbf{Y}_{k}\right\} \\
&=\arg \max _{\left\{s_{p}\right\}_{p=1}^{P} \in \mathscr{C}} \sum_{p=1}^{P}\left\{\operatorname{Re}\left[\operatorname{tr}\left(\mathbf{A}_{p}^{H} \mathbf{Y}_{k-1}^{H} \mathbf{Y}_{k}\right) \bar{s}_{p}\right]\right. \\
&\left.-\operatorname{Re}\left[\operatorname{tr}\left(i \mathbf{B}_{p}^{H} \mathbf{Y}_{k-1}^{H} \mathbf{Y}_{k}\right) \tilde{s}_{p}\right]\right\},
\end{aligned}
$$

which leads to

$$
\begin{aligned}
& \hat{s}_{p}=\arg \max _{s_{p} \in \mathscr{C}}\{ \operatorname{Re}\left[\operatorname{tr}\left(\mathbf{A}_{p}^{H} \mathbf{Y}_{k-1}^{H} \mathbf{Y}_{k}\right) \bar{s}_{p}\right] \\
&\left.-\operatorname{Re}\left[\operatorname{tr}\left(i \mathbf{B}_{p}^{H} \mathbf{Y}_{k-1}^{H} \mathbf{Y}_{k}\right) \tilde{s}_{p}\right]\right\}, \\
& \quad p=1,2, \ldots, P, u=1,2, \ldots, U .
\end{aligned}
$$

\section{ACKNOWLEDGMENTS}

This work was supported in part by the National Science Foundation Grant CCR-0097114 and the Swedish Foundation for Strategic Research (SSF).

\section{REFERENCES}

[1] A. F. Naguib, N. Seshadri, and A. R. Calderbank, "Increasing data rate over wireless channels," IEEE Signal Processing Magazine, vol. 17, no. 3, pp. 76-92, 2000.

[2] S. M. Alamouti, "A simple transmit diversity technique for wireless communications," IEEE Journal on Selected Areas in Communications, vol. 16, no. 8, pp. 1451-1458, 1998.

[3] J. C. Guey, M. P. Fitz, M. R. Bell, and W. Y. Kuo, "Signal design for transmitter diversity wireless communication systems 
over Rayleigh fading channels," in Proc. Vehicular Technology Conference (VTC-98), vol. 1, pp. 136-140, Atlanta, Ga, USA, 1996.

[4] N. Seshadri and J. J. Winters, "Two signaling schemes for improving the error performance of frequency-division-duplex (FDD) transmission systems using transmitter antenna diversity," Int. J. Wireless Inform. Networks, vol. 1, no. 1, pp. 49-60, 1994.

[5] B. L. Hughes, "Differential space-time modulation," IEEE Transactions on Information Theory, vol. 46, no. 7, pp. 25672578, 2000.

[6] J. G. Proakis, Digital Communications, McGraw-Hill, NY, USA, 3rd edition, 1995.

[7] B. M. Hochwald and W. Sweldens, "Differential unitary spacetime modulation," IEEE Transactions on Communications, vol. 48, no. 12, pp. 2041-2052, 2000.

[8] V. Tarokh and H. Jafarkhani, "A differential detection scheme for transmit diversity," IEEE Journal on Selected Areas in Communications, vol. 18, no. 7, pp. 1169-1174, 2000.

[9] J. Liu, J. Li, H. Li, and E. G. Larsson, "Differential space-code modulation for interference suppression," IEEE Trans. Signal Processing, vol. 49, no. 8, pp. 1786-1795, 2001.

[10] G. Ganesan and P. Stoica, "Differential detection based on space-time block codes," to appear in Wireless Personal Communications.

[11] P. Stoica and G. Ganesan, "Maximum-SNR space-time designs for MIMO channels," in Proc. IEEE Int. Conf. Acoustics, Speech, Signal Processing, Salt Lake City, Utah, USA, May 2001.

[12] V. Tarokh, H. Jafarkhani, and A. R. Calderbank, "Space-time block codes from orthogonal designs," IEEE Transactions on Information Theory, vol. 45, no. 5, pp. 1456-1467, 1999.

[13] G. Ganesan and P. Stoica, "Space-time diversity," in Signal Processing Advances in Wireless Communications, G. Giannakis, Y. Hua, P. Stoica, and L. Tong, Eds., Prentice-Hall, NJ, USA, 2000.

[14] Z. Wang and G. B. Giannakis, "Wireless multicarrier communications: where Fourier meets Shannon," IEEE Signal Processing Magazine, vol. 47, no. 3, pp. 29-48, 2000.

[15] G. H. Golub and C. F. Van Loan, Matrix Computations, The John Hopkins University Press, Baltimore, Md, USA, 3rd edition, 1996.

[16] J. C. Liberti Jr. and T. S. Rappaport, Smart Antennas for Wireless Communications: IS-95 and Third Generation CDMA Applications, Prentice Hall PTR, Upper Saddle River, NJ, USA, 1999.

[17] W. C. Jakes Jr., Microwave Mobile Communications, IEEE Press, Piscataway, NJ, USA, 1993.

Jianhua Liu received his B.S. degree from Dalian Maritime University, Dalian, China, in 1984, the M.S. degree from the University of Electronic Science and Technology of China, Chengdu, China, in 1987, and the Ph.D. degree from Tsinghua University, Beijing, China, in 1998, all in Electrical Engineering. From February 1987 to February 1999, he worked at the Communications, Telemetry and Telecontrol Research Institute, Shijiazhuang, China, where he was an Assistant Engineer, Engineer, Senior Engineer, and Research Fellow. From March 1995 to August 1998, he was also a Research Assistant at Tsinghua University. From February 1999 to June 2000, he worked at Nanyang Technological University, Singapore, as a Research Fellow. Since June 2000, he has been a Research Assistant in the Department of Electrical and Computer Engineering at the University of Florida,

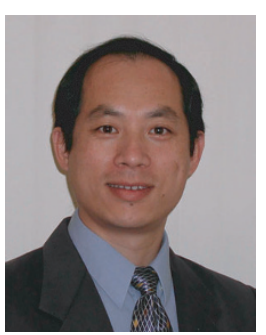

Gainesville, working towards a Ph.D. degree majoring in Electrical Engineering and minoring in Statistics. His research interests include wireless communications, statistical signal processing, and sensor array processing.

Jian Li received the M.Sc. and Ph.D. degrees in electrical engineering from the Ohio State University, Columbus, in 1987 and 1991, respectively. From April 1991 to June 1991, she was an Adjunct Assistant Professor with the Department of Electrical Engineering, the Ohio State University, Columbus. From July 1991 to June 1993, she was an Assistant Professor with the Department of Electrical Engineering, University of Ken-

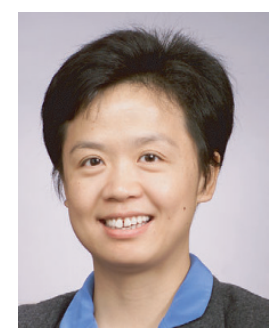
tucky, Lexington. Since August 1993, she has been with the Department of Electrical and Computer Engineering, University of Florida, Gainesville, where she is currently a Professor. Her current research interests include spectral estimation, array signal processing, and signal processing for wireless communications and radar. Dr. Li is a member of Sigma Xi and Phi Kappa Phi. She received the 1994 National Science Foundation Young Investigator Award and the 1996 Office of Naval Research Young Investigator Award. She is currently an Associate Editor for IEEE Transactions on Signal Processing and a Guest Editor for Multidimensional Systems and Signal Processing.

Erik G. Larsson received the M.Sc. degree in Applied Physics and Electrical Engineering from Linköping University, Linköping, Sweden, in 1997. During 1998-99, he was a Research Engineer at Ericsson Radio Systems AB in Stockholm, Sweden, where he was involved in algorithm design and standardization of location services for the GSM system. In 1999 he joined the Department of Systems and Control, Uppsala University,

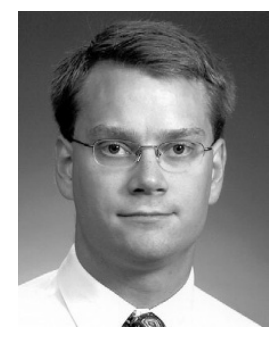
Uppsala, Sweden, where he was a Research and Teaching Assistant and worked towards his Ph.D. degree. Since September 2000, he has been with the Department of Electrical and Computer Engineering, University of Florida, Gainesville, Fla, where he is currently an Assistant Professor. His research interests include wireless communications, statistical signal processing, spectral analysis and radar applications. He has several publications in international journals and holds three U.S. patents. 